Article

\title{
The Unique Immunoregulatory Function of Staphylococcus aureus Lipoteichoic Acid in Dendritic Cells
}

\author{
Suguru Saito ${ }^{1,2,3, *}$, Su-Hui Lin ${ }^{3,4}$ and Hui-Ya $\mathrm{Wu}^{4,5,6}$ \\ 1 Division of Clinical Nephrology and Rheumatology, Graduate School of Medical and Dental Sciences, \\ Kidney Research Center, Niigata University, Niigata 9518510, Japan \\ 2 Department of Bio Medical Science, Cedars-Sinai Medical Center, Los Angeles, CA 90048, USA \\ 3 Institute of Biomedical Science, Academia Sinica, Taipei 115, Taiwan \\ 4 Department of Pathology, Medical College of Georgia, Augusta University, Augusta, GA 30912, USA \\ 5 College of Health Science, Trans World University, Yunlin 640, China \\ 6 Medical Science International, Yamagata 9980811, Japan \\ * Correspondence: suguru.saito@cshs.org; Tel.: +1-706-399-9356
}

Received: 12 June 2019; Accepted: 6 August 2019; Published: 8 August 2019

\begin{abstract}
Background and objectives: Lipoteichoic acid (LTA) is a structural component of Staphylococcus aureus (S. aureus) that induces severe infection disease and skin inflammation such as atopic dermatitis (AD); the biological function of LTA is still unclear. Dendritic cells (DC) are important regulators in the immune system, and the cells ectopically recognize agents that have an influence on the host immune response. We aimed to reveal the DC-based immune response against LTA to understand the novel mechanism in $S$. aureus related acute skin inflammation. Materials and Methods: Different doses of LTA were applied on the epidermal barrier dysfunction mice in order to evaluate the epidermal thickness, DC activation, and subsequent immunological response such as effector T-cell (Teff) activation. In addition, bone marrow-derived dendritic cells (BMDCs) were also treated with LTA, and the immunoregulatory mechanism was investigated. Results: A low dose of LTA did not induce skin inflammation at all; however, a high dose of LTA induced severe skin inflammation on epidermalba rrier dysfunction mice. Those symptoms were correlated with the DC and Teff activation status. The low-dose treatment of LTA showed a suppressive effect in pro-inflammatory cytokine production via a Toll-like receptor 2 (TLR2)-dominant manner, and the effect was significant regarding the co-treatment with another stimulatory signal such as TLR4 by lipopolysaccharide (LPS). Meanwhile, a high-dose treatment of LTA completely abolished the suppressive effect of a low-dose treatment. This phenomenon was based on C-type lectin receptors (CLRs), because the high dose of LTA greatly enhanced the expression of CLRs in the activated DCs. Conclusions: DCs sensed the dose difference of LTA, and the mechanism contributed to regulating immune responses such as effector T-cell activation, which was directly correlated with inflammatory response. This finding might provide an understanding for the novel immunological effect of LTA and S. aureus pathogenesis under inflammation, as well as the mechanism of symbiosis.
\end{abstract}

Keywords: dendritic cell; S. aureus; lipoteichoic acid; toll-like receptor; receptor cross-talking; effector T cell; acute skin inflammation

\section{Introduction}

Staphylococcus aureus (S. aureus) is one of the major commensal bacteria in humans, and it produces several substances [1]. Some of those, such as peptidoglycan (PGN), lipoprotein (LP), and toxins, have been characterized as harmful substances in our body that induce an inflammatory 
response [2,3]. Lipoteichoic acid (LTA) is released from the cell wall of gram-positive bacteria including S. aureus and induces several responses in the host [4,5]. For instance, LTA showed specific influence as a regulator of the inflammatory response [6]. LTA has been described as a ligand of Toll-like receptor 2 (TLR2), and the interaction induced the activation of signaling pathways such as the MyD88 and TRAF complexes in immune cell [7]. Finally, the signal promotes pro-inflammatory cytokine production such as TNF- $\alpha$ and IL-1 $\beta$. However, the LTA function itself is still controversial in the host immunity. LTA induced inflammation bearing with innate immune cell accumulation on the murine skin Intradermal (ID) injection model [8]. On the other hand, naive T cells treated with LTA suppressed the differentiation into effector $\mathrm{T}$ cells by immunological tolerance [9]. Thus, the exact biological and immunological function of LTA is still unclear.

Epidermal barrier dysfunction is the cause of skin inflammation by mechanical stress, and the condition leads to hypersensitivity to several exogenous agents, which aggravate the inflammation [10]. S. aureus tend to be colonized on damaged skin much easier than healthier conditions. As a result, the overgrowing bacteria induces secondary serious inflammation on the skin, such as atopic dermatitis (AD), by specific substances produced from the bacteria [11]. From this point of view, the understanding of the biological function of the bacterial substance is an indispensable part for both prevention and treatment of the bacteria-related inflammation.

Recently, receptor cross-talking was revealed as an important regulative event during inflammation in various cells [12]. TLR signaling is one of the key factors to generate inflammatory responses against ectopic factors not only in immune cells, but also in non-immune cells [13,14]. In general, the TLR signal has been recognized as a unidirectional event to activate inflammatory response; however, it is regulated by another TLR or other receptors under the specific conditions. For instance, TLR3 stimulation with Poly (I:C) was attenuated by LTA through TLR2 [6]. This response strongly suppressed pro-inflammatory cytokine production in keratinocytes. Furthermore, TLR and C-type lectin receptor (CLR) also showed complex inter-regulations as synergistic or suppressive effects on immune cells [15]. Thus, the interaction that is called receptor "cross-talking" is a necessary mechanism to control inflammatory responses.

Here, we showed the unique immunoregulatory mechanism of LTA on dendritic cells (DCs). A low-dose treatment of $S$. aureus LTA interestingly did not induce serious skin inflammation. As an underlying mechanism, the treatment did not activate pro-inflammatory cytokine production on DCs and suppressed effector T cell (Teff) generation. LTA was dominantly recognized by TLR2 in the low-dose treatment, and the signal specifically suppressed another stimulatory signal derived from TLR4 on BMDCs. On the contrary, a high-dose treatment of LTA abolished the suppressive effect on DCs. The high dose of LTA promoted the expression of CLRs (DC-SIGN and Dectin-1) expression dramatically, and the responses strongly induced TNF- $\alpha$ production on bone marrow-derived dendritic cells (BMDCs). It appeared as a synergistic effect of CLRs and TLR2 signaling. Hence, CLR is suspected as a molecular sensor for LTA, which leads to the unidirectional activation of inflammatory response on DCs.

Our finding provides a novel concept of the immunological function of S. aureus-derived LTA in the host immunity. Furthermore, the unique dose-dependent function of LTA may be a clue for an understanding of the mechanism in S. aureus-related inflammation and disease.

\section{Materials and Methods}

\subsection{Mice and Acute Skin Inflammation Model}

C57BL/6J specific pathogen-free (SPF) mice between 8-12 weeks of age were used for the experiment. The dorsal hair was removed the day before start of the experiment. Epidermal barrier dysfunction was induced on the dorsal skin by tape stripping (10 times). The tape-stripped skin was treated with phosphate-buffered saline (PBS), LTA (100 $\mu \mathrm{g}$ for low dose, $1 \mathrm{mg}$ for high dose), and live-S. aureus $\left(10^{6}\right)$ by cotton and cover film. After $72 \mathrm{~h}$ (DC in skin-draining LNs) and $120 \mathrm{~h}$ (epidermal thickness and Teff 
in skin-dLNs) of the treatment, the mice were used for analysis. All the animal experiments were carried out in accordance with the guidelines of the animal welfare committee and the ethics committee of Academia Sinica (IACUC protocol approved No.; 15-10-874, Approved date: 13 October 2016).

\subsection{Reagents and Antibodies}

Lipoteichoic acid (LTA, from S. aureus), phorbol 12-myristate 13-acetate (PMA), lipoprotein lipase (from Pseudomonas sp.) and ionomycin were purchased from Sigma Aldrich (St. Louis, MO, USA). LTA was treated with lipoprotein lipase to inactivate contaminated lipoprotein before using the experiment. A BD Cytofix/Cytoperm ${ }^{\mathrm{TM}}$ Plus Fixation/Permeabilization Solution Kit with BD GolgiStop ${ }^{\mathrm{TM}}$ was purchased from BD Bioscience (Franklin Lakes, NJ, USA). Recombinant murine granulocyte macrophage-colony stimulating factor (rmGM-CSF) and recombinant murine interleukin-4 (rmIL-4) were purchased from Peprotech (Rocky Hill, NJ, USA). Anti-CD45 (30F-11), anti-CD11c (N418), anti-CCR7 (4B12), anti-CD86 (GL-1), anti-CD3 (17A2), anti-CD4 (GK1.5), anti-CD369 (RH1), anti-CD209 (9E9A8), anti-IL-12 (C15.6), and anti-CD16/CD32 (2.4G2) (93) were all purchased from Biolegend (San Diego, CA, USA). Anti-MHC-II (M5/114.15.2), anti-IFN- $\gamma$ (XMG1.2), and erythrocyte lysis buffer were all purchased from Thermo Fisher Scientific (Waltham, MA, USA). The isotype-matched control for each antibody was purchased from the same company. RIPA Lysis and Extraction Buffer, Protease and Phosphatase Inhibitor Cocktail (100X), and phosphate-buffered saline (PBS) were purchased from Thermo Fisher Scientific. PBS was filtrated by a $0.1-\mu \mathrm{m}$ filter to keep the same grade as endotoxin free. The PBS was used for all the experiments in this study. An ECL Western Blotting Detection System was purchased from GE Healthcare Bioscience (Chicago, IL, USA). Anti-TRAF6 (D-10) was purchased from Santa Cruz (Dallas, TX, USA). Anti-NF- $k$ B p65 (D14E12), anti-phospho NF- $k$ B p65 (93H1), anti-p38 MAPK (D13E1), anti-phospho p38 MAPK (D3F9), anti-rabbit IgG, Horseradish peroxidase (HRP)-linked and anti-mouse IgG, and HRP-linked antibody were all purchased from Cell Signaling Technology (Danvers, MA, USA).

\subsection{S. Aureus Culture}

Frozen S. aureus (MRSA; USA300) stock was thawed on ice and then transferred to a tryptic soy broth (TSB; BD bioscience, Franklin Lakes, NJ, USA) and cultured at $37^{\circ} \mathrm{C}$ for $18 \mathrm{~h}$ with shaking. The colony-forming unit (CFU) was calculated in each culture. Heat-killed S. aureus (HK-SA) was prepared with heating at $95^{\circ} \mathrm{C}$ for $30 \mathrm{~min}$. The heated S. aureus suspension was centrifuged at $10,000 \mathrm{rpm}$ for $1 \mathrm{~min}$ to harvest the bacteria cells; then, the cell pellet was resuspended in PBS.

\subsection{Mouse Primary Cell Preparation}

Lymph node cells and splenocytes were obtained from the skin-draining lymph node and spleen respectively by following a method described in a previous report [16]. Briefly, isolated lymph nodes and spleens were crushed on a 70- $\mu \mathrm{m}$ cell strainer; then, the cells were suspended in cell culture medium (RPMI 1640 medium containing with 10\% heat-inactivated fetal bovine serum (FBS), $50 \mathrm{U} / \mathrm{mL}$ of penicillin, $50 \mu \mathrm{g} / \mathrm{mL}$ of streptomycin, and $4 \mathrm{mM}$ of L-glutamine). After being washed once with the cell culture medium, the cells were resuspended in RBC lysis buffer and kept at room temperature (RT) for $5 \mathrm{~min}$. After being washed twice with cell culture medium, the cells were used as lymph node cells and splenocytes. Mouse bone marrow leukocytes were obtained from the tibia and femur by following a method described in a previous report [17]. After extraction of the tibia and femur, bone marrow leukocytes were flushed out with a syringe containing cell culture medium. The cell suspension was filtered through a 70- $\mu \mathrm{m}$ cell strainer and washed once with cell culture medium; then, the cells were treated with RBC lysis buffer at RT for $5 \mathrm{~min}$. After being washed twice with cell culture medium, the cells were used as bone marrow leukocytes. Naïve CD4+T cells were isolated from the splenocyte by using a MagniSort ${ }^{\mathrm{TM}}$ mouse CD4 naïve T cell Enrichment kit (Thermo Fisher Scientific, Waltham, MA, USA). The entire procedure for the cell isolation was performed by following the manual. For intracellular cytokine detection by flow cytometry, some cells 
were stimulated with $100 \mathrm{ng} / \mathrm{mL}$ of PMA and $1 \mu \mathrm{g} / \mathrm{mL}$ of ionomycin or anti-CD3/CD28 antibody under inhibition of protein transportation with GolgiStop ${ }^{\mathrm{TM}}$.

\subsection{Mouse Bone Marrow-Derived Dendritic Cells (BMDCs) Preparation}

Mouse BMDCs were prepared by following a method described in a previous report [17]. At day 0 , $2.0 \times 10^{6}$ of bone marrow leukocytes were suspended in $10 \mathrm{~mL}$ of DC culture medium (cell culture medium containing $20 \mathrm{ng} / \mathrm{mL}$ of rmGM-CSF and rmIL-4), and the cells were seeded on a 100-mm cell culture dish. At day 3,10 mL of fresh DC culture medium was added to the cultured cells. At days 6 and 8, half of the cultured medium was collected and centrifuged; then, the cell pellets were resuspended into $10 \mathrm{~mL}$ of the fresh DC culture medium. The cell suspension was put back into the original plate. At day 10, the cells were used for the experiment.

\subsection{BMDC Stimulation Assay}

Naïve BMDCs were seeded on a 6-well plate with DC culture medium; then, the cells were stimulated by LTA $(10 \mathrm{ng}-10 \mu \mathrm{g} / \mathrm{mL})$, LPS $(100 \mathrm{ng} / \mathrm{mL})$, live-S. aureus $\left(1 \times 10^{6}\right)$, and heat-killed (HK)-S. aureus $\left(1 \times 10^{6}\right)$ for $24 \mathrm{~h}$. In some experiments, TLR2 and CLRs (DC-SIGN, Dectin-1) on the DCs were blocked by anti-TLR2 monoclonal antibody $(\mathrm{mAB})(10 \mu \mathrm{g} / \mathrm{mL})$, anti-DC-SIGN mAB $(10 \mu \mathrm{g} / \mathrm{mL})$, and anti-Dectin-1 $\mathrm{mAb}(10 \mu \mathrm{g} / \mathrm{mL})$, respectively. The efficiency of blocking $\mathrm{mAb}$ was evaluated by the suppression of cytokine production (Supplementary Figure S2). Stimulated cells were analyzed by flow cytometry, and cultured medium was harvested for the measurement of cytokine production by ELISA.

\subsection{In Vitro Antigen Presentation Assay}

Naïve BMDCs were pulsed with ovalbumin (OVA) $(100 \mu \mathrm{g} / \mathrm{mL})$ at $37^{\circ} \mathrm{C}$ for $24 \mathrm{~h}$. In some cultures, LPS $(100 \mathrm{ng} / \mathrm{mL})$ and LTA $(100 \mathrm{ng} / \mathrm{mL})$ were also added by single or combination. Naive or ligand stimulated BMDCs were used for negative (non-antigen) control. The pulsed DCs were mixed with splenic naïve $\mathrm{CD} 4+\mathrm{T}$ cell (DC:T $=1: 10$ ), and then cultured at $37^{\circ} \mathrm{C}$ for $72 \mathrm{~h}$. In the last $6 \mathrm{~h}$ of the culture, proliferated $\mathrm{T}$ cells were re-stimulated with an anti-CD3/CD28 antibody under the inhibition of protein transportation with GolgiStop ${ }^{\mathrm{TM}}$. The proliferated $\mathrm{T}$ cells were stained with fluorochrome-conjugated antibody for both extracellular markers and intracellular cytokines. The stained cells were analyzed by flow cytometry.

\subsection{Flow Cytometry}

Cell surface markers and intracellular cytokines were analyzed by flow cytometers (FACSCanto and LSRII; BD Biosciences, Franklin Lakes, NJ, USA) with the fluorochrome-conjugated monoclonal antibodies described in reagents and antibodies. The cells were initially incubated with FcR blocker (anti-CD16/CD32) at $4{ }^{\circ} \mathrm{C}$ for $10 \mathrm{~min}$. For cell surface marker staining, the cells were incubated with the antibody at $4{ }^{\circ} \mathrm{C}$ for $30 \mathrm{~min}$. Intracellular cytokine staining was performed by using BD Cytofix/Cytoperm ${ }^{\mathrm{TM}}$ Plus Fixation/Permeabilization Solution Kit with BD GolgiStop ${ }^{\mathrm{TM}}$ by following the manual. The dead cells were excluded by forward scatter, side scatter, and propidium iodide gating. All the data were analyzed by BD FACS Diva (BD bioscience, Franklin Lakes, NJ, USA) or FlowJo (Tree Star, Ashland, OR, USA).

\subsection{Sodium Dodecyl Sulfate-Poly Acrylamide Gel Electrophoresis (SDS-PAGE) and Western Blot}

Cell lysates were prepared by treating the cells with $1 \times$ RIPA buffer supplemented with protease inhibitor and phosphatase inhibitor cocktail. The cell lysates were kept at $4{ }^{\circ} \mathrm{C}$ for $30 \mathrm{~min}$, and were then were sonicated a few times. The samples were centrifuged at 12,000 rpm for $30 \mathrm{~min}$; then, the supernatants were harvested as an extracted protein sample. The protein concentration in the samples were measured by bicinchoninic acid (BCA) protein assay. The protein amount in the samples was adjusted with 
SDS sample buffer ( $2 \%$ SDS, $62.5 \mathrm{mM}$ of Tris- $\mathrm{HCl}$ (pH 6.8), $10 \%$ glycerol, $0.01 \%$ bromophenol blue, $50 \mathrm{mM}$ of dithiothreitol (DTT). The protein samples $(20 \mu \mathrm{g})$ were size-separated by SDS-PAGE, and were electronically transferred onto a poly vinylidene difluoride (PVDF) membrane. The membrane was incubated with TBS-T ( $20 \mathrm{mM}$ of Tris- $\mathrm{HCl}$ (pH 7.6), $150 \mathrm{mM}$ of $\mathrm{NaCl}, 0.1 \%$ Tween 20 ) containing $5 \%$ skim milk at room temperature for $1 \mathrm{~h}$, and was further incubated with the primary antibody at $4{ }^{\circ} \mathrm{C}$ for overnight. After being washed with TBS-T for $30 \mathrm{~min}(15 \mathrm{~min} \times 2$ times, the membranes were incubated with the secondary antibody at RT for $30 \mathrm{~min}$. After being washed with TBS-T for $45 \mathrm{~min}$ (15 $\min \times 3$ times), the proteins recognized by the antibody on the membrane were visualized using the ECL Western Blotting Detection System (GE Healthcare Bioscience).

\subsection{TNF- $\alpha$ Measurement by Enzyme-Linked Immuno Sorbent Assay (ELISA)}

TNF- $\alpha$ produced from the stimulated cells was measured by using a TNF- $\alpha$ Mouse ELISA kit (Thermo Fisher Scientific, Waltham, MA, USA). The entire procedure was performed by following the manual.

\subsection{Statistical Analyses}

A Student's $t$-test was used to analyze the data for significant differences. Values of ${ }^{*} p<0.05$ and ${ }^{* *} p<0.01$ were regarded as significant.

\section{Results}

\subsection{Low Dose of S. Aureus LTA is not Harmful to Skin}

We were interested in a dose-dependent effect of $S$. aureus LTA, because prior experiments on a human DC model differentiated by human monocyte cell line THP-1 and on a mouse DC cell line DC 2.4 showed that the treatments with a low dose of LTA $(<100 \mathrm{ng} / \mathrm{mL})$ did not produce pro-inflammatory cytokines. However, the treatments with a high dose of LTA $(>1 \mu \mathrm{g} / \mathrm{mL})$ strongly activated the cells in vitro (unpublished data). The cytokine production did not gradually increase as following the dose of LTA, but it was suddenly upregulated when the dose was over the specific range. Thus, we speculated that LTA might be a trigger in the both immune responses and immunological tolerance with a strict dose-dependent manner on DCs.

We investigated the unique effect of LTA in immune responses on the mice skin using an epidermal barrier dysfunction model, which is a well-established model for the study of the direct interaction with ectopic substance in host skin immunity [18]. The mice were treated by tape stripping, and various agents were applied on the back skin; then, the skin conditions were evaluated after 5 days $(120 \mathrm{~h})$ (Figure 1). A treatment with a low dose of LTA did not induce severe inflammation on the skin, because the superficial condition of the treated area was almost similar to that of the PBS control and healthy (non-treated) skin. On the other hand, a treatment with a high dose of LTA induced severe skin inflammation (Figure 1A). Those differences were also recognized by epidermal thickness in the histological analysis (Figure 1B,C). Although less severe than a live S. aureus treatment, the treatment with a high dose of LTA showed clear epidermal thickness on the skin, and it was more than double that of the skin treated with a low dose. Meanwhile, the skin treated with a low dose of LTA showed almost identical condition to that of healthy (non-treated) skin. Interestingly, the skin treated with PBS showed a slightly increasing of epidermal thickness than the skin treated with a low dose of LTA. The difference was not significant; however, $60 \%$ of the mice $(6 / 10)$ treated with the low dose of LTA showed less thickness in the epidermal layer than PBS-treated mice (unpublished data). 
A
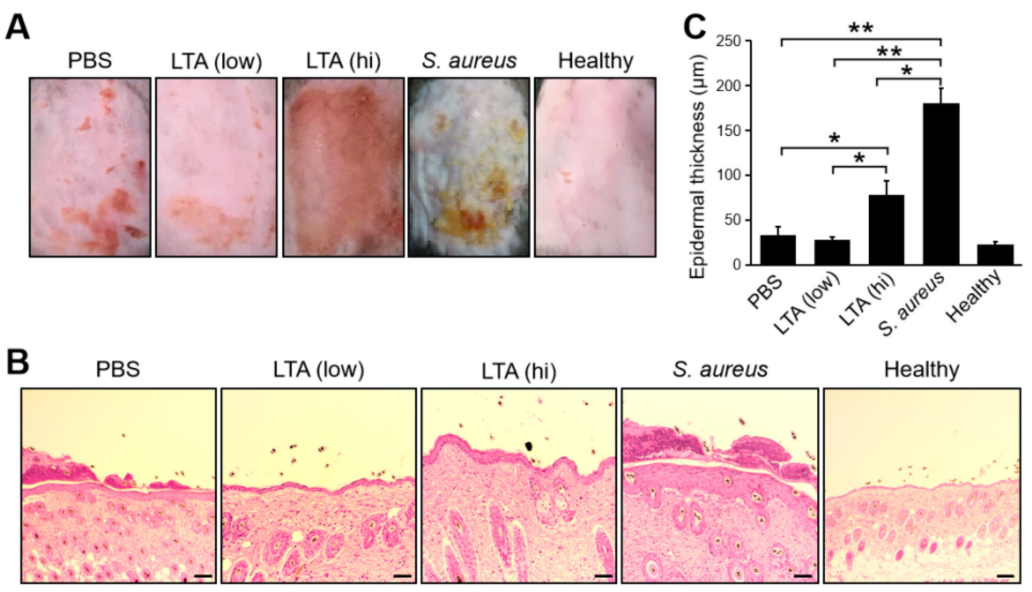

Figure 1. Non-harmful effect of a low dose of lipoteichoic acid (LTA) on epithelial barrier dysfunction mice. (A,B) The superficial and histological images and (C) epidermal thickness on the skin in epidermal barrier dysfunction mice models. Tape-stripped back skins were treated with LTA (100 $\mu \mathrm{g}$ for low dose and $1 \mathrm{mg}$ for high dose), live Staphylococcus aureus (S. aureus) $\left(1 \times 10^{6}\right)$ and PBS (control) respectively. After $120 \mathrm{~h}$ of the treatment, the skin was extracted and used for tissue section Hematoxylin-Eosin stain and epidermal thickness was measured under microscope. The bar indicates $100 \mu \mathrm{m}$. (A-C) Data are representative and pooled from at least five independent animal experiments in each group. The Student's $t$-test was used to analyze data for significant differences. Values of $*<0.01,{ }^{* *} p<0.001$ were regarded as significant.

These results indicate that a treatment with a low dose of LTA is not harmful on the skin; however, a high dose of LTA clearly induces severe skin inflammation.

\subsection{Low Dose of S. Aureus Lipoteichoic Acid Suppresses Dendritic Cell Activation in Acute Skin Inflammation}

On the skin inflammation, epidermal and dermal DCs are regularly activated, and expression levels of major histocompatibility complex (MHC) class II, CD86, and CCR7 are upregulated [19]. In addition, those activated DCs migrate into skin-dLN for generating effector T cells, which is an important player in the inflammatory responses, by the priming effect of DCs to naïve T cells [20]. The acute skin inflammation model showed an increasing of total DCs and migratory DCs (mDCs) in the skin-dLNs of the mice treated with a high dose of LTA and live S. aureus compared with PBS control (Figure 2B,C). On the other hand, the mice treated with a low dose of LTA showed a significantly smaller number of $\mathrm{mDC}$ in the skin-dLNs compared with other mice (Figure 2B,C). The DCs status was correlated with the increasing of CCR7 and CD86 expression levels (Figure 2D,E). IL-12 is a cytokine produced from activated DCs, and it promotes IFN- $\gamma^{+} \mathrm{CD}^{+} \mathrm{T}$ cell (Th1) polarization during $\mathrm{T}$ cell priming in LN. The population of IL-12+DCs in the skin-dLN was also greatly increased in the mice treated with a high dose of LTA and live S. aureus; however, it was significantly decreased in the treatment with a low dose (Figure 2F). The number of Th1 in the skin-dLNs was correlated with DC activation status. The mice treated with a low dose of LTA possessed a significantly smaller number of Th1 compared with the mice treated with a high dose of LTA, live S. aureus, and even PBS control (Figure 2G). 
A

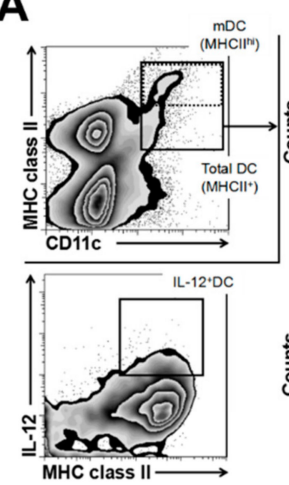

D

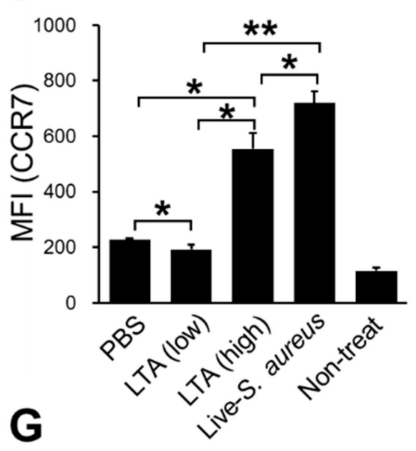

B
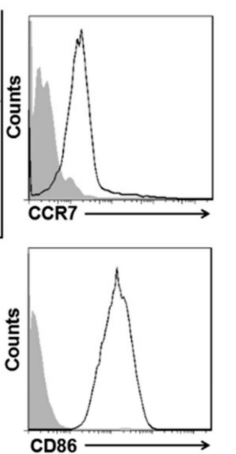

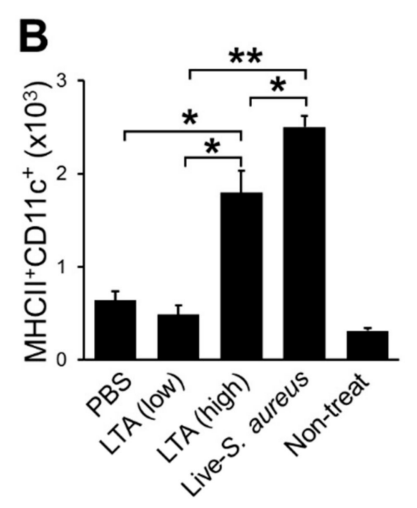

C

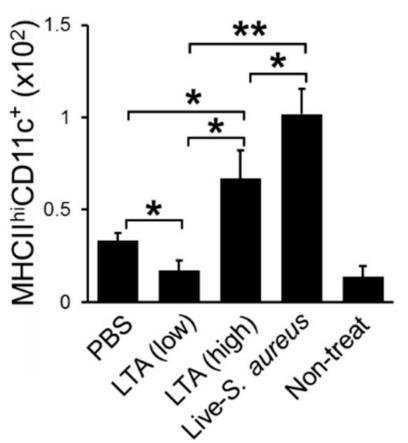

E

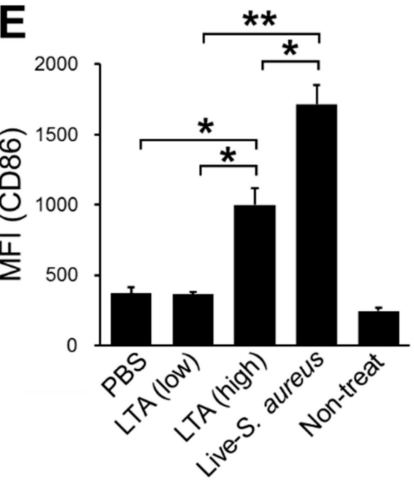

$\mathbf{F}$

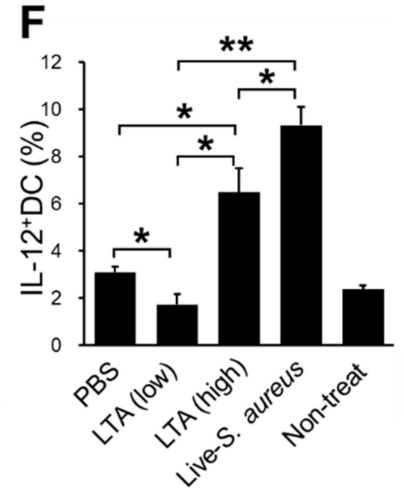

IFN- $\mathrm{\gamma}^{+} \mathrm{CD} 4^{+} \mathrm{T}$ cell $\left(\times 10^{5}\right)$

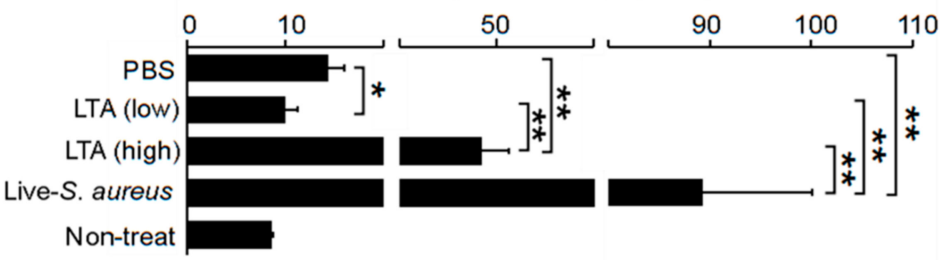

Figure 2. Low dose of LTA attenuates dendritic cells (DC) activation in acute skin inflammation. (A) The gating strategy of DC analysis by flow cytometry. Total DC (MHCII+CD11c+) and migratory DC (MHCIIhiCD11c+) were gated on; then, CCR7 and CD86 expression on the migratory DCs (mDCs) and IL-12 production on total DC was analyzed. (B-G) The mice were treated by tape stripping on the hair removed back skin; then, each substance was applied on the skin. After $72 \mathrm{~h}(\mathbf{B}-\mathbf{F})$ and $120 \mathrm{~h}$ (G) of the treatment, the skin-dLNs were extracted from the mice; then, the isolated cells were used for analysis. (B,C) The number of total DC (B) and $\mathrm{mDC}(\mathbf{C}) .(\mathbf{D}, \mathbf{E})$ The MFI (mean fluorescence intensity) of CCR7 (D) and CD86 (E) expression on mDC. (F) The percentage of IL-12+DC in total DC. (G) Number of IFN- $\gamma^{+} \mathrm{CD}^{+} \mathrm{T}$ cell (Th1) in skin-dLN. (B-F) The positive cells were detected from CD $45^{+}$gated cells. $(\mathbf{G})$ The positive cells were detected from CD45 + CD3+ gated cells. The data are pooled from at least three independent experiments. The Student's $t$-test was used to analyze data for significant differences. Values of ${ }^{*} p<0.05$ and ${ }^{* *} p<0.01$ were regarded as significant.

Taken together, a treatment with a low dose of LTA shows not only a lack of activation, but also an immunosuppressive effect on DCs.

\subsection{LTA Suppresses TLR Mediated DC Activation, However, the Effect Depends on the Dose}

We investigated the mechanism on how LTA regulates the activity of DCs by a dose-dependent manner. The treatments with a low dose of LTA $(0.01,0.1 \mu \mathrm{g} / \mathrm{mL})$ did not activate BMDCs, which was evaluated by TNF- $\alpha$ production (Figure 3A). However, the treatments with a high dose of LTA (0.5-10 $\mu \mathrm{g} / \mathrm{mL})$ absolutely promoted the activation of BMDCs (Figure 3A). Regarding skin inflammation, TLR is frequently activated by several ligands produced from exogenous and endogenous sources [21]. 
The signal is regulated by other signals that are generated from both TLR and another receptor-mediated manner [12]. To mimic the TLR-mediated signal generated under the skin inflammation, we treated BMDCs with LPS. Furthermore, the BMDCs were treated with or without LTA to investigate the immunoregulative effect against TLR signal. Interestingly, the treatments with a low dose of LTA $(0.01,0.1 \mu \mathrm{g} / \mathrm{mL})$ did not activate BMDCs at all (Figure 3A). On the contrary, LTA in the specified dose range significantly suppressed LPS-induced TNF- $\alpha$ production on BMDCs (Figure $3 B$ ). In addition, the combined treatments with LPS and a high dose of LTA $(0.5-10 \mu \mathrm{g} / \mathrm{mL})$ produced more TNF- $\alpha$ than the single treatment with LPS on BMDCs (Figure 3B). We also measured IL-12 production, which is another parameter of DC activation. The response for IL-12 production was similar to TNF- $\alpha$ production in the BMDCs (Supplementary Figure S1).

\section{A}
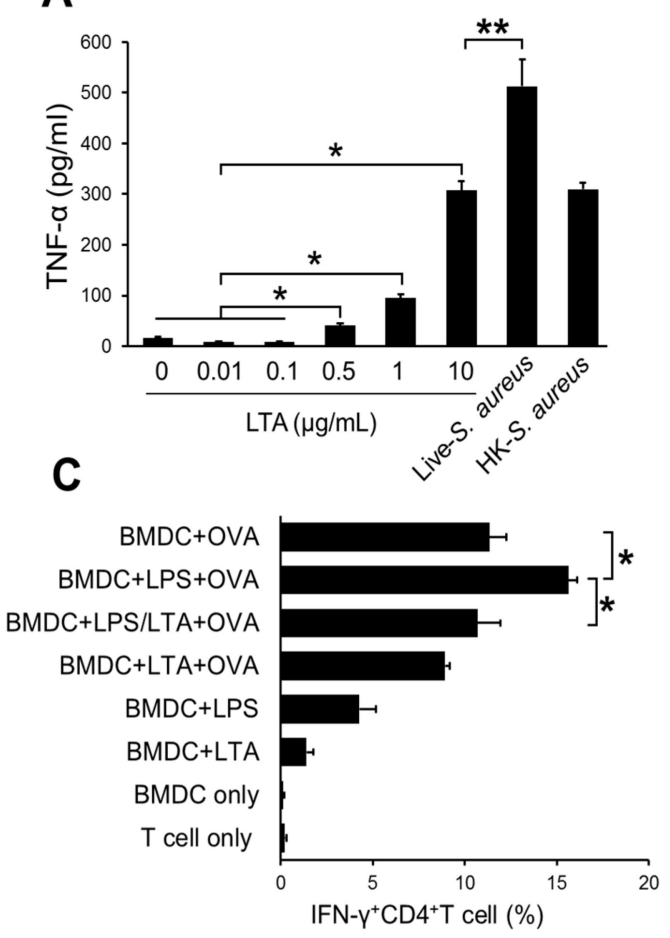

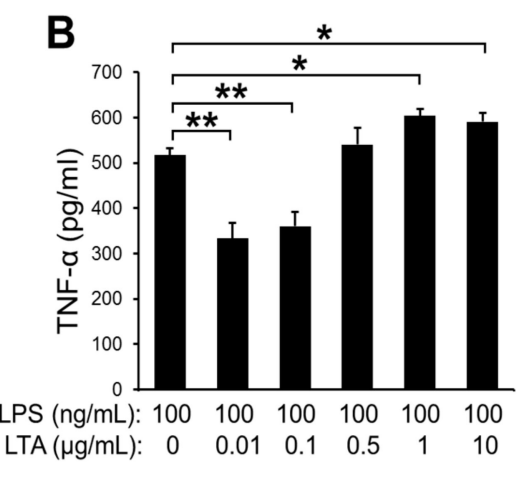

Figure 3. The dose-dependent difference of suppressive effect in LTA treatment. (A) The TNF- $\alpha$ production on bone marrow-derived dendritic cells (BMDCs) with LTA treatment. The BMDCs $\left(1.0 \times 10^{6}\right)$ were treated with LTA. (B) The TNF- $\alpha$ production on BMDCs with LTA and LPS co-treatment. The BMDCs $\left(1.0 \times 10^{6}\right)$ were treated with LTA and LPS. $(\mathbf{A}, \mathbf{B})$ After $24 \mathrm{~h}$ of the treatment, cultured medium was harvested; then, the TNF- $\alpha$ production was measured by ELISA. (C) The differentiation of IFN $-\gamma+\mathrm{CD} 4+\mathrm{T}$ cell (Th1) in antigen presentation assay. The BMDCs were pulsed with OVA $(100 \mu \mathrm{g} / \mathrm{mL}$ ) with or without ligands (LTA: $100 \mathrm{ng} / \mathrm{mL}$, LPS: $100 \mathrm{ng} / \mathrm{mL}$ ) for $24 \mathrm{~h}$. The BMDCs stimulated with only ligands were also prepared for antigen-negative control. The BMDCs were mixed with a naive CD4 + T cell (DC:T = 1:10); then, the cells were incubated for $72 \mathrm{~h}$. The proliferated cells were analyzed, and IFN- $\gamma$-producing cells were detected by flow cytometry. The data are pooled from at least three independent experiments. The Student's $t$-test was used to analyze data for significant differences. Values of ${ }^{*} p<0.05$ and ${ }^{* *} p<0.01$ were regarded as significant.

The activation status of DCs is correlated with antigen-presenting activity in T-cell priming [22]. To investigate the influence of LTA-mediated DCs activation in antigen presentation, we performed an antigen presentation assay. The LPS-stimulated, OVA-pulsed BMDCs generated around $15 \%$ of Th1 cells from naive $\mathrm{CD} 4+\mathrm{T}$ cells by the priming effect (Figure 3C). On the other hand, the OVA-pulsed BMDCs co-treated with LPS and LTA (low dose) showed significantly less percentages of Th1 cell generation (Figure 3C). 
Taken together, a low dose of LTA suppresses the activation of DCs, which is induced by another ectopic stimuli such as the TLR ligand. Furthermore, the suppressive effect of LTA contributes to the attenuation of antigen presentation in naive T-cell priming and effector T-cell generation.

\subsection{TLR Cross-Talking Has Responsibility for the Regulation of DCs Activity}

TLR2 is a receptor for the recognition of $S$. aureus extracellular components such as LTA and peptidoglycan (PGN) [23]. The TLR2 signal originated with LTA has been reported as a unique function in immunosuppression, in which the LTA signal attenuates TLR3-derived pro-inflammatory cytokine production by the TLR cross-talking mechanism in keratinocytes [6]. It was suspected that the same mechanism is working on other cells, including immune cells; therefore, we investigated the TLR2 role in the suppressive effect in a treatment with a low dose of LTA on DCs. The BMDCs stimulated with both LPS and a low dose of LTA showed small amounts of TNF- $\alpha$ production; however, the inhibition of TLR2 by anti-TLR2 mAb canceled the suppressive effect (Figure 4A). The TLR activation induced the upregulation of TRAF6 and downstream transcription factors to promote pro-inflammatory cytokine production [24]. This is a well-conserved pathway for inflammatory responses in immune cells. The BMDCs stimulated with LPS upregulated TRAF6 expression and downstream factors such as phospho-p38 MAPK and Phospho-p65 NF-kB as well (Figure 4B). The combined treatment with LPS and a high dose of LTA showed similar response (Figure 4B), while the treatment with LPS and a low dose of LTA attenuated TRAF6 expression and the expression of downstream transcription factors (Figure 4B). An anti-TLR2 mAb treatment omitted the suppressive effect of the treatment with a low dose of LTA, because TRAF6 and the activation of downstream factors were recovered from the treatment with control IgG (Figure 4B).
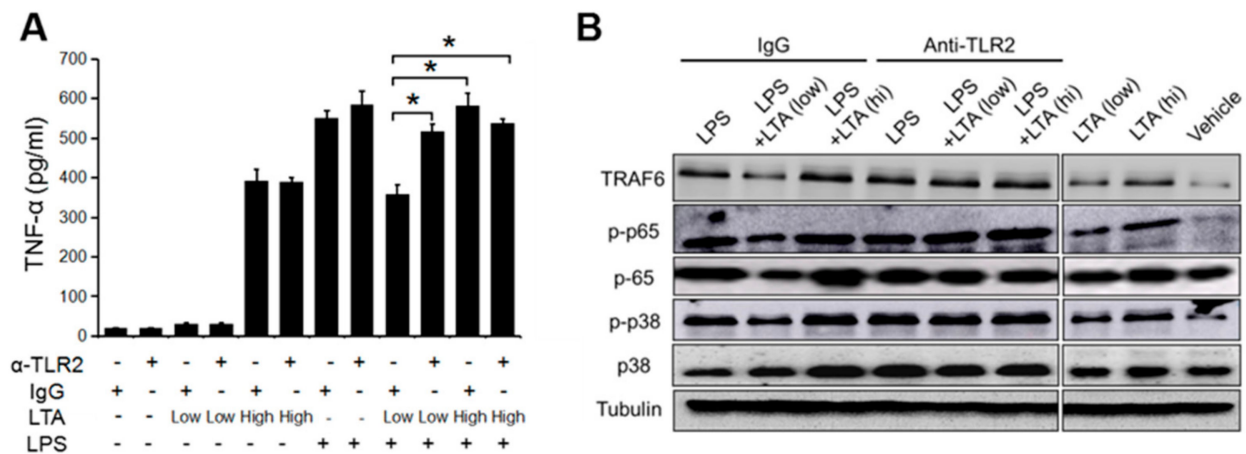

Figure 4. A low dose of LTA suppresses Toll-like receptor 4 (TLR4)-mediated DC activation through TLR2. (A) The TNF- $\alpha$ production on BMDCs with TLR2 inhibition. The BMDCs $\left(1.0 \times 10^{6}\right)$ were treated with LTA (100 ng/mL for low dose and $10 \mu \mathrm{g} / \mathrm{mL}$ for high dose) and LPS (100 ng/mL) with or without anti-TLR2 monoclonal antibody $(\mathrm{mAb})$. After $24 \mathrm{~h}$ of the treatment, the cultured medium was harvested; then, TNF- $\alpha$ production was measured by ELISA. (B) Signaling pathway analysis related with TLR-mediated activation signal on DCs. Cell lysates were prepared from BMDCs from assay (A). Data is pooled from at least three independent experiments (A), and is representative of at least three independent experiments (B). The Student's $t$-test was used to analyze data for significant differences. Values of * $p<0.05$ was regarded as significant.

Thus, the TLR2-madiated LTA signal shows a suppressive effect against TLR4 signal, however, the TLR cross-talking mechanism is only effective in the treatment with a low dose of LTA on DCs.

\subsection{C-Type Lectin Expression is Upregulated by a High Dose of LTA, and the Response is a Trigger} for the Synergistic Effect in DCs Activation

We suspected that the influences of other receptors in the dose-dependent regulation of LTA on DCs. The immune cells including DCs express various pattern recognition receptors (PRRs) such as C-type lectin receptor (CLR) and has a complex function by orchestrating with another PRRs for the controlling 
of specific response $[15,25]$. BMDCs, which were prepared by our system, expressed type I and type II CLR, such as CD369 (Dectin-1, CLEC7A), Dectin-2, CD209 (DC-SIGN), CLEC1A, CD205 (DEC-205), and CD206 (mannose receptor) (unpublished data). The treatment with a high dose of LTA greatly upregulated the expression of DC-SIGN and Dectin-1 on BMDCs, respectively (Figure 5A). The MFI showed that the expression of DC-SIGN and Dectin-1 were significantly increased on the BMDCs treated with a high dose of LTA compared with a low dose of LTA or vehicle control (Figure 5B,C). These responses implied that LTA is recognized by CLRs. The CLR activation and TNF- $\alpha$ production were parallel events, because the treatment with high doses of LTA increased the percentage of TNF- $\alpha$ producing DC-SIGN ${ }^{+}$Dectin- $1^{+}$DCs (Figure 5 D). The blocking of CLRs by mAb suppressed TNF- $\alpha$ production on the BMDCs treated with a high dose of LTA (Figure 5E). Interestingly, the blocking only for TLR2 did not suppress TNF- $\alpha$ production from the BMDC, while the combined blocking of CLRs and TLR2 or independent blocking of CLRs clearly suppressed TNF- $\alpha$ production. To confirm the dominant response of CLR in LTA treatment, we also investigated each function of Dectin-1 or DC-SIGN in LTA-mediated cytokine production. The TNF- $\alpha$ production from LTA-treated BMDC was greatly suppressed by the independent blocking of Dectin-1 and DC-SIGN as well as combined blocking (Supplementary Figure S3).

A
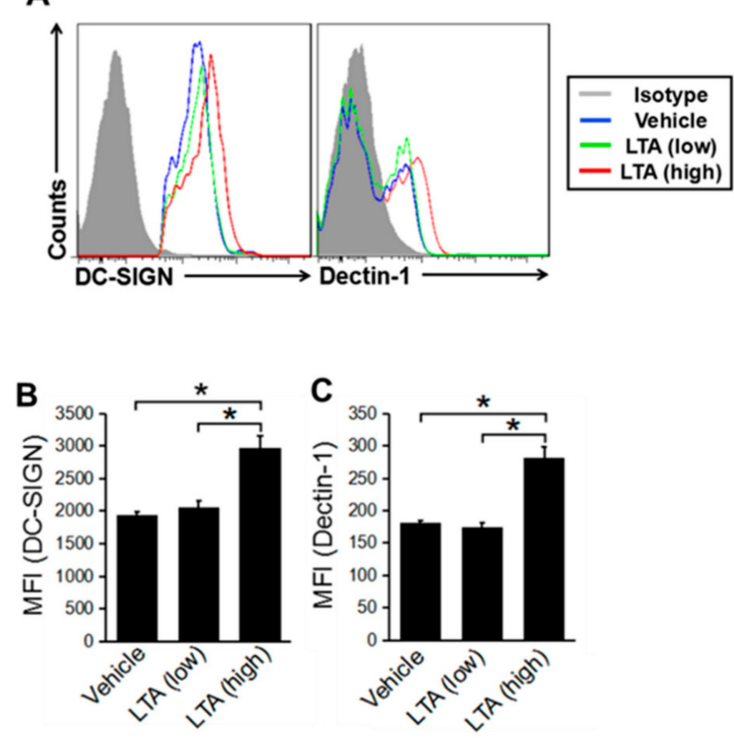

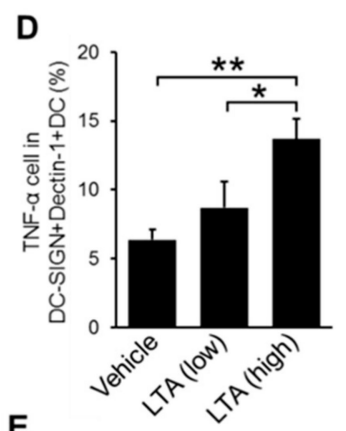

E

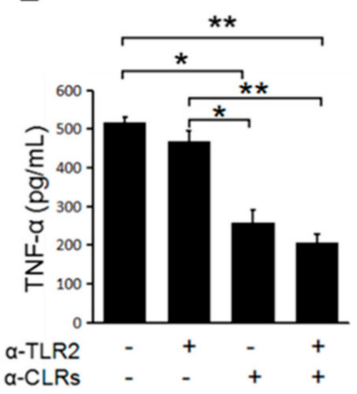

Figure 5. C-type lectin receptors (CLRs) respond to a dose difference of LTA. (A-C) The CLRs' expression change depends on the LTA dose on BMDCs. DC-SIGN and Dectin-1 expression on BMDCs with LTA stimulation ( $100 \mathrm{ng} / \mathrm{mL}$ for low dose and $1 \mu \mathrm{g} / \mathrm{mL}$ for high dose). (A) The MHC class II+CD11c+ cells were gated on; then, DC-SIGN and Dectin-1 expression was detected by flow cytometry. (B,C) The MFI of DC-SIGN and Dectin-1 was calculated from the histogram (A). (D) TNF- $\alpha$ production in CLRs expressed BMDCs. The MHC classII+CD11c+DC-SIGN+Dectin-1+DCs were gated on; then, the TNF- $\alpha^{+}$cells were detected by flow cytometry. (E) TNF- $\alpha$ production from BMDCs with the inhibition of TLR2 and CLRs. BMDCs were stimulated with LTA $(1 \mu \mathrm{g} / \mathrm{mL})$, and TLR2 and CLRs (Dectin-1 and DC-SIGN) were blocked by mAb at the same time. The TNF- $\alpha$ in the cultured medium was measured by ELISA. The data is representative of at least three independent experiments (A), and are pooled from at least three independent experiments (B-E). The Student's $t$-test was used to analyze data for significant differences. Values of $* p<0.05$ and ${ }^{* *} p<0.01$ were regarded as significant. 
Taken together, a high dose of LTA induces CLRs expression on DCs, and it dominantly works for the upregulation of cytokine production in DCs. In addition, CLRs are working as molecular sensors for LTA in a dose-dependent manner in DC activation.

\section{Discussion}

On healthy human skin, S. aureus is not harmful even the bacteria is producing several structural substances including LTA, because the colonization is limited and well controlled by skin homeostasis. However, once the colony was expanded by the specific factors, such as mechanical stress, the host immune system senses the growth, and the response against the bacteria is suddenly activated [26]. Traditionally, this response has been understood as based on the total number of growing bacteria itself [27]. It has been reported that skin-colonized bacteria are able to penetrate the epidermis layer on the seriously wounded skin, which lost barrier function. However, the frequency and absolute number of bacteria in the event are not high [28]. The evidence of direct interaction between non-invasive $S$. aureus and host immune cells-except for the Langerhans cell, which is one of the subsets of skin resident DC-on the inflamed skin layer has been unclear [29]. The total amount of structural substances released from the bacteria may increase on the wounded skin, so that immune cells, such as the DCs and macrophages, have a chance to contact with these substances. In fact, a report showed that the AD skin possessed a larger amount of S. aureus LTA than healthy skin [30]. Thus, we hypothesized that the LTA amount produced by S. aureus on the skin is a critical factor in the activation of the host immune response.

Our result revealed that LTA shows a dose-dependent influence on DCs and the effector T-cells-based immune response in the acute skin inflammation (Figures 2 and 3). Abundant LTA strongly activated DCs, so that the migration into skin-dLNs and cytokine production was increased. As an underlying mechanism in the upregulation of the immune response on the LTA-treated DC, the activation of the TRAF-mediated pathway was detected in our result (Figure 4B). In addition, the expression of CLRs was upregulated by a treatment with high doses of LTA, and the signal generated by the receptors seemed to be working to promote the activation of the DCs (Figure 5). Interestingly, TLR2 expression was not upregulated by LTA treatment; therefore, we thought that CLRs sensed the dose differences of LTA with high sensitivity (unpublished data).

We also found a unique effect of LTA in the immune response based on DCs. The treatment with a low dose of LTA did not activate DCs. On the contrary, the treatment negatively regulated the activation of DCs, which was induced by another receptor-mediated signal such as TLR4 (Figure 3B). The influence of the response was obviously transferred from DCs to the subsequent immune response such as effector T-cell generation (Figures 2 and 3C).

The dose-dependent suppressive effect of LTA was regulated by TLR2 mediated manner on DCs (Figure 4A). This is an unexpected mechanism on DCs from the previous understandings; however, the response was very clear. As a mechanism in this response, we showed that TRAF6-mediated activation of p65 and p38 was regulated by LTA in a dose-dependent manner (Figure 4B). This pathway is well characterized in not only immune cell but also in other cells, so further mechanisms in the LTA-mediated cytokine regulation will be revealed in a future study.

We concluded that the unique immunoregulative effect of LTA is severely correlated with the dose of LTA, and the response is mainly controlled by CLR (Figures 5 and 6). Since CLRs expression levels on DCs were upregulated in the treatment with a high dose of LTA (Figure 5A,B), the CLRs can be thought of as a molecular sensor for LTA with a dose-dependent manner. In addition, CTRs' blocking greatly suppressed TNF- $\alpha$ production in LTA-simulated BMDC (Figure 5E). In fact, we confirmed that the affinity between LTA and CTL (Dectin-1 and DC-SIGN) was much stronger than between TLR2 by surface plasmon resonance (unpublished data). However, the mechanism through which DC recognizes the alteration of the LTA dose in the natural environment has still been unclear. Further investigation will be required to address these points. In addition, we suspected that not only CLRs but also another receptor senses the LTA dose difference on DCs. The dose-dependent 
immunoregulative effect of LTA may provide a good understanding for the cause of $S$. aureus-related skin inflammation. On healthy skin, $S$. aureus might have a beneficial effect by producing a moderate amount of LTA, which keeps the skin in a healthy condition. The concept can explain the mechanism of the balance between the symbiosis and pathogenesis of commensal bacteria in the human body.

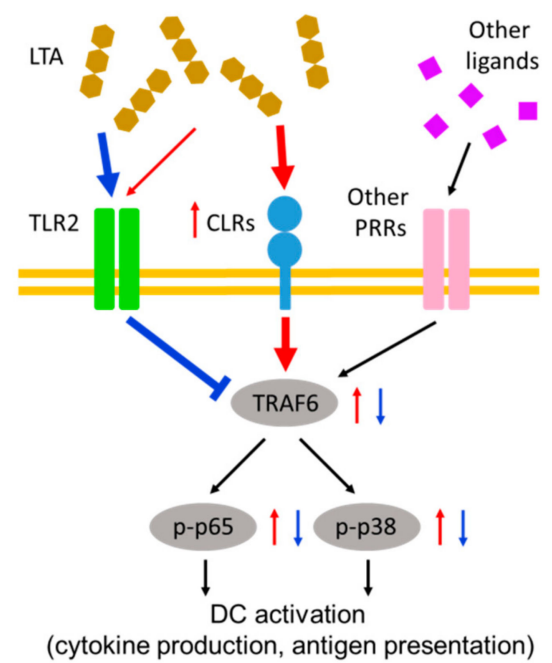

Figure 6. Immunoregulatory effect of LTA-mediated receptor cross-talking on DCs. In a low dose treatment with LTA, DCs are not activated at all. On the contrary, the stimulation signal from another receptor is suppressed by the LTA signal through TLR2. A high dose treatment with LTA induces an increasing of CLRs expression, and the signal from CLRs coactivates DCs with another receptor signal in a TNF receptor associated factor (TRAF)-mediated manner. In this case, the immunosuppressive effect by the low dose of LTA via TLR2 is completely abolished. The red arrows indicate the response in a high dose treatment with LTA. The blue arrows and line indicate the response in a low dose treatment with LTA.

\section{Conclusions}

This study showed a novel concept of an amount-dependent response against LTA on DCs. $\mathrm{DC}$ is an immune regulator, and it controls $\mathrm{T}$ cell activity by antigen presentation and cytokine production. Hence, the immunoregulative effect of LTA on DCs may be a key factor to control the T-cell-based inflammatory response, including acute skin inflammation. LTA is produced from live bacteria contentiously during their growing, and it is released from bacterial cells, even after being killed or inactivated. Therefore, LTA may be a therapeutic target in gram-positive bacteria related disease and inflammation. In addition, the function of LTA has a possibility to reveal the reason why symbiosis is established in our immune system. A low amount of LTA did not induce DCs and T-cell activation in our results. On the contrary, the treatment suppressed another stimulatory signal on DCs. These are interesting findings and clues for the understanding of the strategy of how to control bacterial growth to maintain a healthy environment and prevent inflammation in our body.

Supplementary Materials: The following are available online at http://www.mdpi.com/2571-8800/2/3/22/ s1, Figure S1: IL-12 production from BMDC was suppressed by LTA with dose dependent manner, Figure S2: The efficiency of TLR2, Dectin-1 and DC-SIGN blocking antibodies, Figure S3: Cytokine production was dominantly suppressed by CLRs inhibition in BMDC.

Author Contributions: Conceptualization, S.S. and S.-H.L.; Methodology, S.S. and S.-H.L.; Software, S.S. and H.-Y.W.; Validation, S.S., S.-H.L. and H.-Y.W.; Formal analysis, S.S., S.-H.L. and H.-Y.W.; Investigation, S.S., S.-H.L. and H.-Y.W; Resources, S.S.; Data curation, S.S., S.-H.L. and H.-Y.W.; Writing-Original draft preparation, S.S. and S.-H.L.; Writing-Review and editing, S.S. and S.-H.L.; Visualization, S.S., S.-H.L. and H.-Y.W.; Supervision, S.S.; Project administration, S.S.; Funding acquisition, S.S.

Funding: This research was funded by JSPS, Shounai Fund of Young Human Resource Development and Shounai midori group. 
Acknowledgments: We thank Shu-Heng Lin and Ming-Yu Hsieh for the discussion and technical assistance. We also thank Silvia Mayle Leanhart for the assistance in manuscript writing.

Conflicts of Interest: The authors declare no conflict of interest.

\section{References}

1. Krismer, B.; Weidenmaier, C.; Zipperer, A.; Peschel, A. The commensal lifestyle of Staphylococcus aureus and its interactions with the nasal microbiota. Nat. Rev. Microbiol. 2017, 15, 675-687. [CrossRef] [PubMed]

2. Nguyen, M.T.; Götz, F. Lipoproteins of Gram-Positive Bacteria: Key Players in the Immune Response and Virulence. Microbiol. Mol. Biol. Rev. 2016, 80, 891-903. [CrossRef]

3. De Marzi, M.C.; Todone, M.; Ganem, M.B.; Wang, Q.; Mariuzza, R.A.; Fernández, M.M.; Malchiodi, E.L. Peptidoglycan recognition protein-peptidoglycan complexes increase monocyte/macrophage activation and enhance the inflammatory response. Immunology 2015, 145, 429-442. [CrossRef]

4. Rismondo, J.; Percy, M.G.; Gründling, A. Discovery of genes required for lipoteichoic acid glycosylation predicts two distinct mechanisms for wall teichoic acid glycosylation. J. Biol. Chem. 2018, 293, 3293-3306. [CrossRef] [PubMed]

5. Breyne, K.; Steenbrugge, J.; Demeyere, K.; Vanden Berghe, T.; Meyer, E. Preconditioning with Lipopolysaccharide or Lipoteichoic Acid Protects against Staphylococcus aureus Mammary Infection in Mice. Front. Immunol. 2017, 8, 833. [CrossRef] [PubMed]

6. Lai, Y.; Di Nardo, A.; Nakatsuji, T.; Leichtle, A.; Yang, Y.; Cogen, A.L.; Wu, Z.R.; Hooper, L.V.; Schmidt, R.R.; Von Aulock, S.; et al. Commensal bacteria regulate Toll-like receptor 3-dependent inflammation after skin injury. Nat. Med. 2009, 15, 1377-1382. [CrossRef]

7. Oliveira-Nascimento, L.; Massari, P.; Wetzler, L.M. The Role of TLR2 in Infection and Immunity. Front. Immunol. 2012, 3, 79. [CrossRef]

8. Wada, K.; Fujii, E.; Ishida, H.; Yoshioka, T.; Muraki, T. Effect of lipoteichoic acid on dermal vascular permeability in mice. J. Pharmacol. Exp. Ther. 2000, 294, 280-286. [PubMed]

9. Kaesler, S.; Skabytska, Y.; Chen, K.M.; Kempf, W.E.; Volz, T.; Köberle, M.; Wölbing, F.; Hein, U.; Hartung, T.; Kirschning, C.; et al. Staphylococcus aureus-derived lipoteichoic acid induces temporary T-cell paralysis independent of Toll-like receptor 2. J. Allergy Clin. Immunol. 2016, 138, 780-790.e6. [CrossRef]

10. De Benedetto, A.; Kubo, A.; Beck, L.A. Skin Barrier Disfunction-A Requirement for Allergen Sensitization? J. Investig. Dermatol. 2012, 132, 949-963. [CrossRef] [PubMed]

11. Paharik, A.E.; Parlet, C.P.; Chung, N.; Todd, D.A.; Rodriguez, E.I.; Van Dyke, M.J.; Cech, N.B.; Horswill, A.R. Coagulase-Negative Staphylococcal Strain Prevents Staphylococcus aureus Colonization and Skin Infection by Blocking Quorum Sensing. Cell Host Microbe 2017, 22, 746-756.e5. [CrossRef] [PubMed]

12. Latz, E.; Golenbock, D.T. Receptor “cross talk" in innate immunity. J. Clin. Investig. 2003, 112, $1136-1137$. [CrossRef] [PubMed]

13. Dasu, M.R.; Thangappan, R.K.; Bourgette, A.; DiPietro, L.A.; Isseroff, R.; Jialal, I. TLR2 expression and signaling-dependent inflammation impair wound healing in diabetic mice. Lab Investig. 2010, 90, 1628-1636. [CrossRef] [PubMed]

14. Yu, L.; Wang, L.; Chen, S. Endogenous toll-like receptor ligands and their biological significance. J. Cell. Mol. Med. 2010, 14, 2592-2603. [CrossRef] [PubMed]

15. Ostrop, J.; Lang, R. Contact, Collaboration, and Conflict: Signal Integration of Syk-Coupled C-Type Lectin Receptors. J. Immunol. 2017, 198, 1403-1414. [CrossRef] [PubMed]

16. Saito, S.; Kawamura, T.; Higuchi, M.; Kobayashi, T.; Yoshita-Takahashi, M.; Yamazaki, M.; Abe, M.; Sakimura, K.; Kanda, Y.; Kawamura, H.; et al. RASAL3, a novel hematopoietic RasGAP protein, regulates the number and functions of NKT cells. Eur. J. Immunol. 2015, 45, 1512-1523. [CrossRef]

17. Saito, S.; Quadery, A.F. Staphylococcus aureus Lipoprotein Induces Skin Inflammation, Accompanied with IFN- $\gamma$-Producing T Cell Accumulation through Dermal Dendritic Cells. Pathogens 2018, 7, 64. [CrossRef]

18. Liu, H.; Archer, N.K.; Dillen, C.A.; Wang, Y.; Ashbaugh, A.G.; Ortines, R.V.; Kao, T.; Lee, S.K.; Cai, S.S.; Miller, R.J.; et al. Staphylococcus aureus Epicutaneous Exposure Drives Skin Inflammation via IL-36-Mediated T Cell Responses. Cell Host Microbe 2017, 22, 653-666.e5. [CrossRef] 
19. Shklovskaya, E.; Roediger, B.; De St. Groth, B.F. Epidermal and Dermal Dendritic Cells Display Differential Activation and Migratory Behavior While Sharing the Ability to Stimulate CD4+ T Cell Proliferation In Vivo. J. Immunol. 2008, 181, 418-430. [CrossRef]

20. PTordesillas, L.; Lozano-Ojalvo, D.; Dunkin, D.; Mondoulet, L.; Agudo, J.; Merad, M.; Sampson, H.A.; Berin, M.C. DL2+ CD11b+ dermal dendritic cells capture topical antigen through hair follicles to prime LAP+ Tregs. Nat. Commun. 2018, 9, 5238. [CrossRef]

21. Chen, L.; Guo, S.; Ranzer, M.J.; DiPietro, L.A. Toll-like receptor 4 plays an essential role in early skin wound healing. J. Investig. Dermatol. 2013, 133, 258-267. [CrossRef] [PubMed]

22. Langenkamp, A.; Messi, M.; Lanzavecchia, A.; Sallusto, F. Kinetics of dendritic cell activation: Impact on priming of TH1, TH2 and nonpolarized T cells. Nat. Immunol. 2000, 1, 311-316. [CrossRef] [PubMed]

23. Wang, Z.; MacLeod, D.T.; Di Nardo, A. Commensal bacteria lipoteichoic acid increases skin mast cell antimicrobial activity against vaccinia viruses. J. Immunol. 2012, 189, 1551-1558. [CrossRef] [PubMed]

24. Kobayashi, T.; Walsh, P.T.; Walsh, M.C.; Speirs, K.M.; Chiffoleau, E.; King, C.G.; Hancock, W.W.; Caamano, J.H.; Hunter, C.A.; Scott, P.; et al. TRAF6 is a critical factor for dendritic cell maturation and development. Immunity 2003, 19, 353-363. [CrossRef]

25. Wevers, B.A.; Kaptein, T.M.; Zijlstra-Willems, E.M.; Theelen, B.; Boekhout, T.; Geijtenbeek, T.B.; Gringhuis, S.I. Fungal engagement of the C-type lectin mincle suppresses dectin-1-induced antifungal immunity. Cell Host Microbe 2014, 15, 494-505. [CrossRef]

26. Brown, A.F.; Leech, J.M.; Rogers, T.R.; McLoughlin, R.M. Staphylococcus aureus Colonization: Modulation of Host Immune Response and Impact on Human Vaccine Design. Front. Immunol. 2013, 4, 507. [CrossRef]

27. Nakatsuji, T.; Chen, T.H.; Narala, S.; Chun, K.A.; Two, A.M.; Yun, T.; Shafiq, F.; Kotol, P.F.; Bouslimani, A.; Melnik, A.V.; et al. Antimicrobials from human skin commensal bacteria protect against Staphylococcus aureus and are deficient in atopic dermatitis. Sci. Transl. Med. 2017, 9, eaah4680. [CrossRef] [PubMed]

28. Nakatsuji, T.; Chen, T.H.; Two, A.M.; Chun, K.A.; Narala, S.; Geha, R.S.; Hata, T.R.; Gallo, R.L. Staphylococcus aureus Exploits Epidermal Barrier Defects in Atopic Dermatitis to Trigger Cytokine Expression. J. Investig. Dermatol. 2016, 136, 2192-2200. [CrossRef]

29. Van Dalen, R.; De La Cruz Diaz, J.S.; Rumpret, M.; Fuchsberger, F.F.; Van Teijlingen, N.H.; Hanske, J.; Rademacher, C.; Geijtenbeek, T.B.H.; Van Strijp, J.A.G.; Weidenmaier, C.; et al. LC capture Staphylococcus aureus wall teichoic acid is a pathogen-associated molecular pattern that is recognized by langerin (CD207) on skin Langerhans cells. bioRxiv 2017. [CrossRef]

30. Travers, J.B.; Kozman, A.; Mousdicas, N.; Saha, C.; Landis, M.; Al-Hassani, M.; Yao, W.; Yao, Y.; Hyatt, A.M.; Sheehan, M.P.; et al. Infected atopic dermatitis lesions contain pharmacologic amounts of lipoteichoic acid. J. Allergy Clin. Immunol. 2010, 125, 146-152.e1-2. [CrossRef] 